\title{
ACOMPANHAMENTO AMBULATORIAL DE PACIENTES COM MIELOMENINGOCELE EM UM HOSPITAL PEDIÁTRICO
}

\author{
J. FRANCISCO SALOMÃO*, RENÊ D. LEIBINGER*, JOÃO G. S. CARVALHO*, \\ JOSÉ ALVARO B. PINHEIRO*, GIANNE L. LUCCHESI*, VERA BOMFIM**
}

\begin{abstract}
RESUMO - A mielomeningocele constitui a mais frequente malformação congênita do sistema nervoso e, a despeito de sua complexidade e acometimento de múltiplos órgãos, é compatível a sobrevida prolongada. Por esses motivos, o acompanhamento de pacientes com essa malformação assume vital importância no que diz respeito a qualidade de sua sobrevida. Com o objetivo de avaliar a qualidade do acompanhamento de portadores de spina bifida cística tratados em um hospital pediátrico, 54 pacientes foram examinados e seus familiares entrevistados. A inexistência de um centro multidisciplinar de tratamento de portadores de defeito de fechamento do tubo neural na cidade do Rio de Janeiro obrigou os pacientes a se deslocarem para locais geograficamente distantes entre si em busca de tratamento complementar em várias especialidades correlatas, com reflexos negativos na qualidade de sobrevida. Desta maneira, apenas uma quarta parte dos pacientes era capaz de se locomover e a chamada deambulação comunitária era praticamente nenhuma. Apenas $2 / 3$ se submetiam regularmente a fisioterapia e apenas a metade era regulamente acompanhada por ortopedistas. Quase $50 \%$ dos pacientes não foram orientados a procurar assistência urologica e $75 \%$ apresentavam incontinência urinária. A incidência de infecçōes urinárias de repetição foi $72,2 \%$. As complicações e intercorrências neurocirúrgicas propriamente ditas tiveram incidências comparáveis às observadas na literatura. Concluímos que a qualidade de sobrevida dos pacientes estudados é significativamente afetada por fatores sócio-económicos e pela ausência de centros multidisciplinares de tratamento.
\end{abstract}

PALAVRAS-CHAVE: spina bifida, mielomeningocele, disrafismo espinhal, clínica multidisciplinar.

\section{Follow-up of myelomeningocele patients in the outclinic of a pediatric hospital}

SUMMARY - Myelomeningocele is the most common congenital malformation of the nervous system and despite its complexity and involvement of multiple organs is compatible with long survival. The peculiar characteristics of this malformation expose myelomeningocele patients to acute and chronic care problems with effects in quality of survival. In order to evaluate the quality of the follow-up of spina bifida patients in a pediatric hospital, the authors examined 54 patients attending the neurosurgical outpatient unity of a pediatric hospital. The lack of a multidisciplinary spina bifida clinic in Rio de Janeiro forced the patients to pursuit for complimentary medical and paramedical care outside the hospital with significant effects in the quality of survival. In consequence, only $25 \%$ of the patients were able to walk and community ambulation was nearly absent. Only $66.6 \%$ had a regular rehabilitation program and nearly $50 \%$ had routine orthopedics consultations. Almost half of the patients had no urological referral at all and $75 \%$ were incontinent, with recurrent urinary infections ranging $72.2 \%$. The rates of neurosurgical complications were similar to those observed in the literature. We concluded that the quality of survival of patients with neural tube defects is strongly influenced by the adverse socio-economical conditions and the lack of a spina bifida multidisciplinary clinic.

KEY WORDS: Spina bifida, neural tube defects, myelomeningocele, multidisciplinary clinic.

Serviço de Neurocirurgia Pediátrica, Departamento de Cirurgia*, Departamento de Pediatria**, Instituto Fernandes Figueira (M.S. - Fiocruz), Rio de Janeiro, RJ. Aceite: 7-janeiro-1995. 
A mielomeningocele é a mais complexa de todas as malformações congênitas do sistema nervoso central (SNC) e, caracteristicamente, se associa a múltiplas anomalias do encéfalo, medula espinhal, nervos periféricos e sistema ósteo-articular. A despeito de sua complexidade, é compatível a sobrevida prolongada e o acompanhamento de pacientes com esse defeito assume grande importância, visto que: a) a maioria tem QI normal ou próximo ao normal ${ }^{11,14}$; b) em sua maioria, exibem quadros de paralisias de membros inferiores em graus variáveis, muitas vezes atenuados pelo uso de aparelhos ortopédicos apropriados e sessōes intensivas de reabilitaçāo ${ }^{2,11}$; ) quase todos são portadores de distúrbios esfincterianos, vesicais e anais, de difícil controle, predisponentes a infecçōes urinárias e altamente limitantes do ponto de vista médico e social ${ }^{1,11,15}$; d) em mais de $70 \%$ dos casos há hidrocefalia associada e, deste modo, estão potencialmente sujeitos a deterioração neurológica consequente a disfunção do sistema de drenagem ${ }^{11.16}$; e) são igualmente sujeitos a deterioração neurológica devido a patologias associadas (malformação de Chiari do tipo II, hidrosiringomielia e síndrome da medula ancorada; entre outras) $)^{7.11}$; f) em virtude da pouca mobilidade são propensos a obesidade ${ }^{6,16} ; \mathrm{g}$ ) em decorrência de todos os aspectos expostos, são particularmente propensos a sindromes de inadaptação social ${ }^{4}$.

A necessidade de oferecer um melhor atendimento, orientado às reais necessidades destes pacientes, levou-nos a analisar a qualidade do atendimento prestado aos portadores de mielomeningocele acompanhados no ambulatório do Serviço de Neurocirurgia do Instituto Fernandes Figueira da Fundação Oswaldo Cruz, Rio de Janeiro, RJ (IFF-Fiocruz).

\section{CASUÍSTICA E MÉTODOS}

Foram revistos os prontuários dos pacientes com mielomeningocele tratados no IFF-Fiocruz, entre janeiro1987 e dezembro-1993. Os familiares foram convocados para entrevistas, por telegrama ou telefone, e os pacientes examinados por um único neurocirurgião. De uma população inicial de 72 pacientes cujos prontuários foram localizados, $6(8,3 \%)$ faleceram no período inicial de internação, a maioria no contexto de alteraçôes relacionadas à malformação de Chiari do tipo II. Outros 5 faleceram no período de acompanhamento, a maioria $(n=4)$ devido a disfunçōes do sistema de derivação ventricular previamente implantado. Destes, apenas 2, por ocasião do óbito, foram assistidos na Instituiçāo. Sete pacientes não foram localizados. Deste modo, pode-se admitir a mortalidade mínima de $15,2 \%$ (11 pacientes sabidamente falecidos) e máxima de $25 \%$, caso se venha a supor que todos os perdidos para acompanhamento tenham morrido.

$\mathrm{O}$ atual estudo, assim sendo, é baseado em 54 pacientes com idade variando entre 1 e 13 anos (média 4,85 anos) (Fig 1), sendo $37(68,5 \%)$ do sexo feminino e $17(31,5 \%)$ do sexo masculino; $45(83,3 \%)$ eram portadores de derivaçōes ventriculares para controle da hidrocefalia.

\section{RESULTADOS}

Motivo de acompanhamento irregular: dos 54 pacientes, $43(79,6 \%)$ são regularmente acompanhados no ambulatório de neurocirurgia, não tendo faltado a qualquer consulta no período de 1 ano, contado até a data da entrevista. As alegações dos familiares de $11(20,3 \%)$

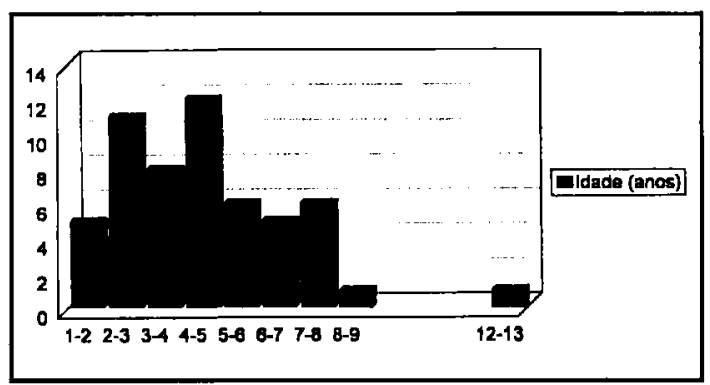

Fig 1. Distribuição dos 54 pacienter por faixa etária pacientes com frequência irregular ao ambulatório têm como fundamentos: a longa distância a percorrer desde suas residências até o Hospital $(n=4)$; as características da própria doença, que dificultam a locomoção do paciente $(n=3)$; a falta de tempo para atender às consultas agendadas $(n=2)$; problemas de natureza financeira $(n=2)$. Todas estas alegações, a nosso ver, são reflexo da precária situação econômica dos entrevistados. 


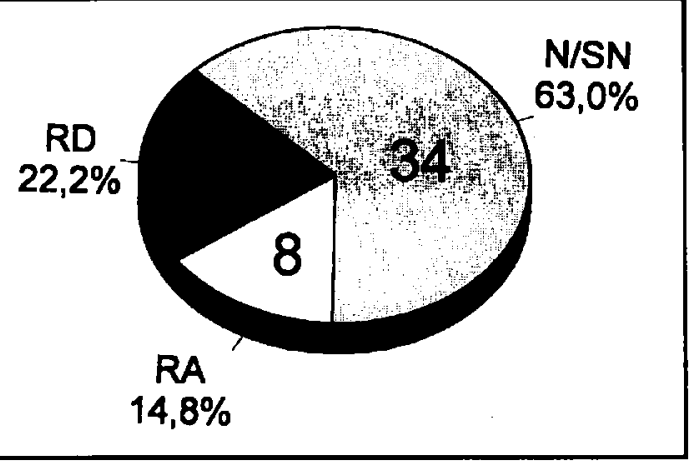

Fig 2. Capacidade intelectual medida pelo Teste de Desenvolvimento de Denver: $N / S N$, normal ou subnormal; $R D$, retardo discreto; $R A$, retardo acentuado.
Avaliação do desempenho intelectual: a dificuldade em se obter em curto espaço de tempo uma avaliação mais fina do desempenho intelectual destes pacientes, bem como as limitaçōes impostas pela idade (maioria na faixa dos 4 anos de vida), levou-nos a optar pela aplicação do Teste de Desenvolvimento de Denver (TDD): 34 pacientes $(62,9 \%)$ tiveram desenvolvimento considerado normal ou próximo ao normal, de acordo com a faixa etária, $8(14,8 \%)$ foram considerados portadores de retardo mental importante (Fig 2). De 27 crianças cm idade escolar, $18(66,6 \%)$ frequentam escolas, 3 delas em estabelecimentos especializados para deficientes; 34 crianças nunca frequentaram qualquer tipo de escola (27 devido à baixa faixa etária) e 2 abandonaram qualquer tipo de atividade discente devido à falta de adaptação.

Função motora: 10 pacientes (18,5\%) não apresentavam, por ocasião da avaliação, nenhum tipo de restrição motora; $20(37 \%)$ eram paraplégicos; $19(35,2 \%)$ tinham paraparesia em grau variável; $4(7,4 \%)$ tinham monoplegia ou monoparesia de um membro inferior. Apenas 14 pacientes deambulavam (25,9\%) (Fig 3). O índice de deambulação comunitária, ou seja, locomoção favorecida pelo uso de aparelhos ortopédicos, foi praticamente nulo nesta amostra, o que permite considerar que os pacientes que deambulavam eram os que não apresentavam nenhum impedimento maior para esta função e, em sua maioria, independiam de qualquer tipo de órtese.

Fisioterapia: 36 pacientes $(66,6 \%)$ submetiam-se regularmente a fisioterapia e reabilitação; $10(18,5 \%)$ haviam abandonado o tratamento motivados, em sua maioria, pelas longas distâncias entre os centros de tratamento e suas residências; 7 (12,9\%) pacientes nunca tiveram qualquer assistência fisiátrica, a maioria devido ao acentuado retardo psicomotor $\mathrm{e}$ intelectual.

Função esfincteriana e acompanhamento urológico: apenas 4 pacientes $(7,4 \%)$ detinham total controle da função esfincteriana; apenas $6(11,1 \%)$ eram socialmente continentes, ou scja, tinham a capacidade de se manterem secos por períodos de 3 a 4 h em função de manobras urológicas ou medicamentos; 41 (75,9\%) eram totalmente incontinentes (Fig 4). A metade dos pacientes ( $n=27$ ) não tinha acompanhamento urológico ou este era feito de maneira irregular. Deste total, $12(44,4 \%)$ nunca foram adequadamente orientados a procurar assistência urológica. Esta realidade se refletiu no alto índice de infecções urinárias de repetição observado $(n=39$, ou 72,2\%).

\section{Acompanhamento ortopédico:}

31 pacientes $(57,4 \%)$, submetiam-se regularmente a tratamento ortopédico; $12(22,2 \%)$ tiveram o tratamento interrompido, dentre os quais 7 com alta por decisão médica e 5 por dificuldade de acesso aos locais de tratamento; 11 pacientes $(20,4 \%)$ jamais procuraram qualquer tipo de tratamento ortopédico, a maioria alegando dificuldade de acesso aos locais de tratamento indicados.

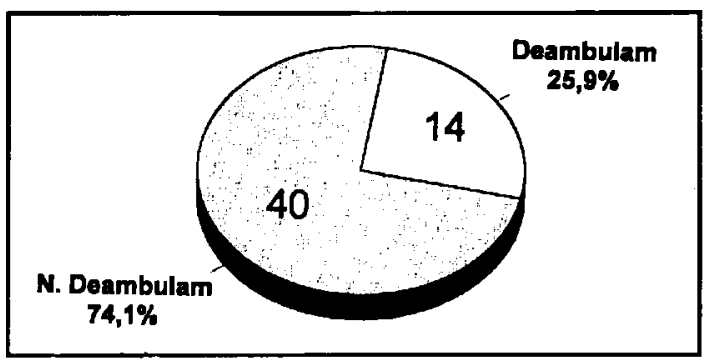

Fig 3. Capacidade de deambulaçāo. 


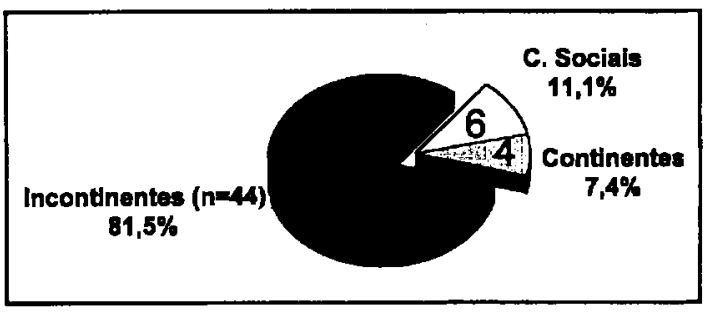

Fig 4. Função urológica em 54 pacientes avaliados. Note-se o baixo indice de continência social.
Complicaf̧ões relacionadas à hidrocefalia: de $45(83,3 \%)$ pacientes com derivaçöes ventriculares para controle da hidrocefalia, $17(37,7 \%)$ apresentaram complicações, sendo a maioria decorrente de disfunções mecânicas do sistema de drenagem ( $\mathrm{n}=11$ ou $24,4 \%$ ). Complicações infecciosas aconteceram em 4 pacientes $(8,8 \%)$, incidência compatível com os índices globais da Instituição.

Malformação de Chiari do tipo II: sinais e sintomas a ela relacionados ocorreram em apenas 2 pacientes $(3,7 \%)$, na vigência de disfunção de derivação e, em ambos, a sintomatologia regrediu após normalização da pressão intracraniana. Nenhum caso de síndrome da medula ancorada foi diagnosticado na presente série.

Avaliação da situação pelos familiares: 48 familiares $(88,9 \%)$ informaram que, se necessário repetiriam tudo o que até agora havia sido feito pelos pacientes e que o esforço por eles dispendido fora compensador; 27 (50\%) confidenciaram que interromperiam uma eventual futura gestação, caso viessem a ter conhecimento de um novo caso de mielomeningocele; $21(38,9 \%)$, levariam a gestação a termo, por motivos de ordem moral ou religiosa.

\section{COMENTÁRIOS}

Existe um crescente consenso de que pacientes com mielomeningoceles devam ser tratados de maneira agressiva e que o tratamento é capaz de beneficiar a maioria dos acometidos pela malformação ${ }^{12,15,16}$. Esta constataçāo simplesmente reflete a evoluçāo dos conceitos relacionados à postura médica frente a portadores de mielomeningocele, não mais se justificando a adoção de critérios seletivos, conforme os propostos pelo grupo de Sheffield há aproximadamente 20 anos $^{10}$. A análise de uma série de 407 casos de mielomeningocele publicada por Laurence, em 1964", revela que o número de pacientes que teve o defeito corrigido correspondia a pouco mais de uma terça parte da população estudada, que a maioria morria de infecções intracranianas e que a possibilidade de um recém-nascido alcançar os 12 anos de idade era de $29 \%$. A introdução de antibióticos mais potentes, bem como o desenvolvimento e posterior aperfeiçoamento dos sistemas de derivação ventricular, aumentou consideravelmente os indices de sobrevivência destes pacientes, sendo parcialmente responsáveis pela popularização dos chamados critérios de seleção, cujo objetivo era oferecer tratamento apenas àqueles pacientes considerados de bom prognóstico. Desta maneira, a coincidência de paraplegia, hidrocefalia volumosa, mielocifose e outros defeitos congênitos associados era considerada de mau prognóstico e responsabilizadas pelo baixo desempenho intelectual que estes pacientes apresentavam ${ }^{10}$. A adoção de critérios seletivos pelo grupo de Sheffield ${ }^{10}$ resultou em óbito de todos os pacientes não elegíveis para tratamento cirürgico em um período de 9 meses, resultados não reproduzidos por outros autores ${ }^{12}$. A análise de séries não selecionadas veio, posteriormente, demonstrar que: a) o maior limitativo ao desempenho intelectual dos pacientes não era a hidrocefalia, mas a ocorrência de infecções doSNC e hemorragia intracerebral ${ }^{11,14,15,18 ;}$; ) as taxas de mortalidade de séries selecionadas e não selecionadas não apresentavam diferenças significativas ${ }^{11,13,15}$. Estas constataçōes levaram a maioria dos centros médicos a abandonar os critérios seletivos, oferecendo tratamento a todos os portadores do defeito, exceção feita àqueles em condiçōes agônicas ou pré-agônicas por ocasião do nascimento.

O reconhecimento da complexidade desta patologia e a constatação de que portadores de mielomeningocele podem apresentar, concomitantemente, problemas agudos e crônicos com repercussão em múltiplos aparelhos e sistemas, levou à implantação, em vários países, de centros 
multidisciplinares de tratamento de portadores de spina bifida, incontestes responsáveis pela melhoria na qualidade de sobrevida dos pacientes ${ }^{12}$. Um exemplo recente ${ }^{8}$ revela que, após a desativação de um destes centros, os pacientes passaram a ser irregularmente acompanhados, com reflexos significativos no aumento da morbidade e na redução da qualidade de sobrevida, bem como no elevado número de cirurgias potencialmente evitáveis (como úlceras de decúbito e nefrectomias), caso se mantivesse acompanhamento regular. É importante ressaltar que a mortalidade dos portadores de mielomeningocele não se estabiliza após os 2 primeiros anos de vida e que, embora em menor proporção, óbitos continuam ocorrendo. Na série de McLone ${ }^{13}$, a maioria destes óbitos se deu após procedimentos cirírgicos de natureza diversa, possivelmente relacionados a disfunçōes não detectadas de derivações. Na nossa série, pudemos observar que as mortes foram em grande número associadas a disfunçōes de sistemas de derivação, com a maioria dos pacientes atendidos em outros hospitais onde, provavelmente, estas complicações não tenham sido reconhecidas e tratadas adequadamente.

Na presente série estudam-se pacientes acompanhados em um hospital pediátrico do Rio de Janeiro, cuja clientela é oriunda de estratos populacionais desfavorecidos. A cidade não dispõe, a exemplo da maioria cidades do País, de um Centro de Referência Multidisciplinar de Tratamento de Spina Bifida (CRMT), sendo possível especular que a situação por nós encontrada não difere significativamente da observada na maioria dos estados brasileiros. A ausência de CRMT obriga os familiares a procurarem recursos de tratamento complementar (como ortopédico, fisiátrico e urológico) em pontos geograficamente distantes entre si e a análise dos motivos alegados para a frequência irregular aos ambulatórios, na realidade, indica como responsáveis problemas de natureza sócioeconômica relacionados, principalmente, à locomoção e ao transporte destes pacientes. Esta situação condena importante percentual de crianças à ausência de qualquer forma de tratamento fisiátrico e ortopédico, com reflexos no baixo índice de deambulaçāo comunitária, que na série de Chicago corresponde a $75 \%$ dos sobreviventes e a $60 \%$ da população inicial de 100 crianças estudadas ${ }^{13,15}$. Diversos estudos ${ }^{5.12 .13 .16}$ demonstram que, à medida que a criança cresce, a capacidade de deambulação comunitária tende a diminuir, em função do aumento do peso corporal. Nossos dados demonstram que apenas $25 \%$ dos pacientes desta série eram capazes de deambular e que a maioria dos que detinham esta função (10/14) a desempenhavam por não apresentarem qualquer impedimento maior. Seguramente, este número poderia ser bem maior $e$ beneficiar grande percentual de pacientes dotados de algum tipo de motilidade nos membros inferiores, caso lhes fosse oferecido tratamento ortopédico adequados.

Uma situação ainda mais preocupante relaciona-se à função urológica. $O$ número de pacientes que atinge a chamada continência social mostrou-se bastante reduzido $(\mathrm{n}=6$ ou $11,1 \%)$, contrastando com os índices apresentados pelo grupo de Chicago, superiores a $80 \%{ }^{13.15}$. Esta situação tem importantes repercussões sobre a função renal, com infecçōes urinárias de repetição observadas em aproximadamente três quartos dos nossos pacientes. Outro agravante decorre de que aproximadamente $50 \%$ dos pacientes não foram adequadamente orientados a procurar assistência urológica. É importante também ressaltar que a incontinência urinária, presente em $75,9 \%$ dos pacientes desta série, constitui importante limitativo de ordem social.

Caso se compare o desempenho intelectual de nossos pacientes com o registrado em outras séries ${ }^{1,13-13}$, não observamos alterações significativas entre elas, embora os métodos por nós utilizados para aferir estes parâmetros sejam até certo ponto subjetivos, em função das várias limitações impostas à realização dos testes. Os relatos da literatura ${ }^{3.7 .13,15}$ revelam que $25 \%$ dos pacientes sobrevivem em condições de absoluta falta de competitividade, imposta pelo baixo QI. Os $75 \%$ restantes têm inteligência normal ou proxima ao normal. Apesar destes dados, admite-se que as dificuldades de aprendizado sejam bastante elevadas neste grupo ${ }^{13}$. É necessário ressaltar que a maioria dos pacientes por nós estudados se encontra em faixas etárias bastante baixas, o que poderá, no futuro, reduzir os índices atualmente apresentados. 
Tabela 1. Dados comparativos de pacientes com mielomeningocele sem acompanhamento nas diversas especialidades em três clínicas de neurocirurgia pediátrica com características diversas.

\begin{tabular}{lccc}
\hline & $\begin{array}{c}\text { St. Louis Children's } \\
\text { Hospital, St. Louis, Mo }\end{array}$ & $\begin{array}{c}\text { Children's Memorial } \\
\text { Hospital, Chicago, III }\end{array}$ & $\begin{array}{c}\text { IFF - Fiocruz } \\
\text { Rio de Janeiro, RJ }\end{array}$ \\
\hline Especialidades & & & \\
Ortopedia & $50 \%$ & $25 \%$ & $30 \%$ \\
Urologia & $45 \%$ & $30 \%$ & $50 \%$ \\
Neurocirurgin & $66 \%$ & $22 \%$ & $20 \%$ \\
\hline
\end{tabular}

Em relação às complicações oriundas da presença de derivações ventriculares, nossos índices não diferem dos observados em hidrocefalias de qualquer etiologia $e$ a incidência de infecções, ao contrário do observado quando da instalação do "shunt" inicial, encontra-se de acordo com a média do nosso Serviço, para hidrocefalias de diversas etiologias ${ }^{17}$. Importante citar que a maioria dos portadores de mielomeningocele que deterioram tardiamente, o fazem em consequência de disfunçōes de sistemas de derivação e que a falência desses sistemas pode se manifestar não somente por síndrome de hipertensão intracraniana mas, também, por escoliose, hidrosiringomielia ou sintomas relacionados à junção bulbo-cervical no contexto da malformação de Chiari do tipo II ${ }^{7,11}$, conforme observado em alguns pacientes da presente série.

Os dados referentes ao acompanhamento de nossos pacientes foram comparados aos apresentados por Kaufman e col. ${ }^{\mathrm{R}}$ após a desativação da clínica de defeitos congênitos de St. Louis, Mo, e aos observados no Children's Memorial Hospital de Chicago, III ${ }^{8,13}$, que possui uma clínica multidisciplinar bastante atuante (Tabela 1). Observamos que nossos dados se encontram mais próximos aos de Chicago do que dos de St. Louis, principalmente no que se refere a atendimento neurocirúrgico e ortopédico. No entanto, é preciso considerar a diferença na média de idade entre nossos pacientes e os da série de Chicago, cujo tempo de acompanhamento já ultrapassa 15 anos $^{13}$. É sabido que, à medida que os pacientes avançam em idade, a freqüência a consultas diminui, dentre outros motivos devido à dificuldade de locomoção e transporte aos locais de acompanhamento. Por apresentarem média de idade bastante inferior à do grupo de Chicago, tomou-se menos penoso transportar os pacientes sob nossos cuidados até os locais de atendimento, motivo pelo qual os índices de frequência aos ambulatórios de neurocirurgia e ortopedia, são quase superponíveis. Por motivos já expostos, observamos elevada incidência de pacientes sem acompanhamento urológico. Em nosso julgamento, à medida que nossos pacientes avançarem em anos, haverá um absenteísmo progressivamente crescente, caso mantido o atual "status-quo", com significativo percentual de abandono de tratamento, caso năo se criem centros multidisciplinares de tratamento e acompanhamento para portadores de spina bifida.

É interessante notar que a grande maioria dos familiares por nós entrevistados demonstrou surpreendente capacidade de conviver com os diversos aspectos da doença. A importância deste dado $€$ ainda mais relevante quando consideramos que eles são, em sua grande maioria, oriundos das camadas mais desfavorecidas de nossa sociedade, residindo longe dos locais de tratamento e, consequentemente, com dificuldade de acesso aos mesmos. Conforme demonstrado em nosso inquérito, a grande maioria nāo se arrependeu da decisão de autorizar o tratamento da criança e, caso neçessário fosse, repetiria as ações realizadas.

\section{CONCLUSŌES}

1) No mínimo $75 \%$ dos pacientes inicialmente tratados em nossa instituição sobreviveu ao primeiro ano de vida e encontra-se em tratamento ambulatorial.

2) A ocorrência de hidrocefalia na presente série foi de $83,3 \%$ e sua existência, responsável pela maioria dos óbitos observados no acompanhamento.

3) Mais de 60\% dos pacientes têm desempenho intelectual normal ou próximo à normalidade. 
4) O percentual de pacientes que deambula é acentuadamente inferior ao registrado na literatura internacional e reflete as deficiências do tratamento complementar oferecido.

5) Há alto índice de pacientes desassistidos no que se refere a acompanhamento fisiátrico, ortopédico e urológico.

6) A qualidade de sobrevida é significativamente afetada por fatores sócio-econômicos e pela ausência de centros multidisciplinares de acompanhamento e tratamento.

\section{REFERÊNCIAS}

1. Bailey RB. Urologic management of spina bifida. In Rekate HL (ed). Comprehensive management of spina bifida, Boca Raton CRC Press: 1991, p 185-213.

2. Carrol N. The orthopedic and orthotic management of the spina bifida child. Clin Neurosurg 1983, 30:413-435.

3. Charney E. Weller S, Sutton L, Bruce DA. Managment of the newborn with meningomyelocele: time for a decision making process. Pediatrics 1985, 75:58-64.

4. Dallyn L, Garrison-Jones C. Reaching adulthood: the long-term psychosocial adjustment of children with spina bifida. In Rekate HL (ed). Comprehensive management of spina bifida, Boca Raton: CRC Press, 1991, p 215-237.

5. Dias $\mathrm{L}$. The orthopedic aspects of spinal dysraphism. In Raimondi AJ, Choux M, Di Rocco C (eds). The Pediatric spine, Vol 1. (Development and the dysraphic state).New York: Springer, 1989, p 210-219.

6. Dixon MS, Rekate HL. Pediatric management of children with myelodysplasia. In Rekate HL (ed). Comprehensive management of spina bifida, Boca Raton: CRC Press,1991,. p 49-65.

7. Epstein F. Meningomyelocele: "pitfalls" in early and late management. Clin Neurosurg 1983, 30:366-383.

8. Kaufman BA, Terbrock A, Winters N, Ito J, Klosterman A, Park TS. Disbanding a multidisciplinary clinic: effects on the healt care of myelomeningocele patients. Pediatr Neurosurg 1994, 21:36-44.

9. Laurence KM. The natural history of spina bifida cistica: detailed analysis of 407 cases. Arch Dis Child 1964, 39:41.

10. Lorber J. Results of treatment of myelomeningocele: an analysis of 524 unselected cases whith special reference to possible selecion for treatment. Dev Med Child Neurol 1971, 13:279-303.

11. McLone DG. Results of treatment of children born with a myelomeningocele. Clin Neurosurg 1983, 30: 407-435.

12. McLone DG. Tratment of myelomeningocele: arguments against selection. Clin Neurosurg 1986, 33:359-370.

13. McLone DG. Continuing concepts in management of spina bifida. Pediatr Neurosurg 1992, 18:254-256.

14 McLone DG, Czyzewski D, Raimondi AJ, Sommers RC. Central nervous system infections as a limiting factor in the intelligence of children with meningomyelocele. Pediatrics 1982, 70:338-342.

15. McLone DG, Naidich. Myelomeningocele : outcome and late complications. In McLaurin RL, Schut $\mathrm{L}$, Venes JL, Epstein F (eds). Pediatric neurosurgery: surgery of the developing nervous system Ed 2. Philadelphia: Saunders, 1989, p 53-70.

16. Rekate HL. Neurosurgical management of the newborn with spina bifida. In Rekate HL (ed). Comprehensive management of spina bifida. Boca Raton: CRC Press, 1991, p 28.

17. Salomão JF, Leibinger RD, Menezes MLB, Carvalho JGS - Infecção nas derivações ventriculares em uma populaçăo pediátrica. Arq Bras Neurocirurg 1993, 12:255-264.

18. Soare PL, Raimondi AJ. Intellectual and perceptual motor characteristics of treated myelomeningocele children. Am J Dis Child 1977, 131:199-204. 